\title{
Diffusion and segmental dynamics of rodlike molecules by fluorescence correlation spectroscopy
}

Roland G. Winkler

Citation: The Journal of Chemical Physics 127, 054904 (2007); doi: 10.1063/1.2753160

View online: https://doi.org/10.1063/1.2753160

View Table of Contents: http://aip.scitation.org/toc/jcp/127/5

Published by the American Institute of Physics

\section{Articles you may be interested in}

Dynamic structure factor of semiflexible macromolecules in dilute solution

The Journal of Chemical Physics 104, 6355 (1996); 10.1063/1.471297

Internal dynamics of semiflexible polymers with active noise

The Journal of Chemical Physics 146, 154903 (2017); 10.1063/1.4981012

Diffusion limited first contact of the ends of a polymer: Comparison of theory with simulation

The Journal of Chemical Physics 105, 3878 (1996); 10.1063/1.472208

Models and equilibrium properties of stiff molecular chains

The Journal of Chemical Physics 101, 8119 (1994); 10.1063/1.468239

Dynamic properties of molecular chains with variable stiffness

The Journal of Chemical Physics 102, 7750 (1995); 10.1063/1.469027

Rotational and translational diffusion of short rodlike molecules in solution: Oligonucleotides

The Journal of Chemical Physics 94, 2324 (1991); 10.1063/1.459904

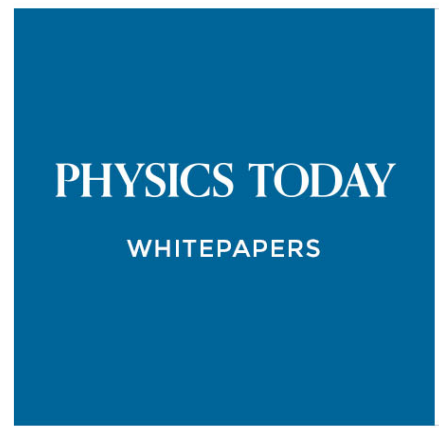

ADVANCED LIGHT CURE ADHESIVES

Take a closer look at what these environmentally friendly adhesive systems can do

\section{READ NOW}

PRESENTED BY

Q MASTERBOND' 


\title{
Diffusion and segmental dynamics of rodlike molecules by fluorescence correlation spectroscopy
}

\author{
Roland G. Winkler ${ }^{\text {a) }}$ \\ Institut für Festkörperforschung, Forschungszentrum Jülich, D-52425 Jülich, Germany
}

(Received 8 May 2007; accepted 5 June 2007; published online 6 August 2007)

\begin{abstract}
The dynamics of weakly bending polymers is analyzed on the basis of a Gaussian semiflexible chain model and the fluorescence correlation spectroscopy (FCS) correlation function is determined. Particular attention is paid to the influence of the rotational motion on the decay of the FCS correlation function. An analytical expression for the correlation function is derived, from which the averaged segmental mean square displacement can be determined independent of any specific model for the polymer dynamcis. The theoretical analysis exhibits a strong dependence of the correlation function on the rotational motion for semiflexible polymers with typical lengths and persistence lengths of actin filaments or fd viruses. Hence, FCS allows for a measurement of the rotational motion of such semiflexible polymers. The theoretical results agree well with experimental measurements on actin filaments and confirm the importance of large relaxation times. (c) 2007 American Institute of Physics. [DOI: 10.1063/1.2753160]
\end{abstract}

\section{INTRODUCTION}

Living organisms comprise a broad spectrum of flexible, semiflexible, and rodlike polymers such as DNA, actin filaments, and microtubules. ${ }^{1}$ The particular conformational degrees of freedom are essential for their biological function and the knowledge of the dynamics is important for predicting and understanding their macroscopic behavior. ${ }^{2,3} \mathrm{~A}$ physical property which often distinguishes biological macromolecules from the majority of synthetic polymers is the presence of bending restrictions on length scales much larger than their thickness. This leads to correlations among segments along a polymer which are characterized by the persistence length $\left(l_{p}\right)$. A number of experimental ${ }^{4-8}$ and theoretical studies ${ }^{9,10}$ show that semiflexibility modifies not only the behavior of molecules with persistence lengths smaller or on the order of the contour length, but also that of seemingly flexible polymers, i.e., polymers with contour lengths much larger than the persistence length. Hence, it is essential to take polymer stiffness adequately into account in theoretical descriptions.

From the experimental point of view, fluorescence correlation spectroscopy (FCS) is very well suited for studying the dynamics of biological macromolecules. ${ }^{11-16}$ By fluorescent labeling of individual segments ${ }^{13,16}$ or continuous labeling of the whole molecule, ${ }^{12,14}$ the diffusional motion of segments as well as that of the overall molecule can be studied at nanomolar concentration under (quasi)equilibrium conditions in solution and cellular systems. For objects significantly smaller than the FCS detection volume, only the overall (center-of-mass) diffusion is measured. For objects larger than the laser focus, however, the intramolecular dynamics

${ }^{a)}$ Electronic mail: r.winkler@fz-juelich.de can be measured in addition. ${ }^{12-16}$ In Ref. 16, we determined center-of-mass diffusion coefficients and the longest relaxation times for double-stranded (ds) DNA molecules and demonstrated that the DNA dynamics can be described by the theory of semiflexible polymers. ${ }^{3,17}$ The persistence length of F-actin filaments $\left(l_{p} \approx 17 \mu m\right)$ is larger than the lateral extension of the FCS detection volume. This provides the opportunity to study the polymer dynamics on length scales below the persistence length. In Ref. 14, we demonstrated that the FCS correlation function can be described by a semiflexible polymer model and in Ref. 15 the segmental mean square displacement has been extracted from the FCS correlation function by a model independent approach. These results confirm various theoretical predictions for the time dependence of the intramolecular dynamics. For ds DNA, we have shown that hydrodynamic interactions control the dynamics $^{14,16}$ in agreement with predictions of Zimm ${ }^{18,19}$ and a semiflexible polymer model. ${ }^{3,17,20}$ (Note, this finding contradicts FCS results presented in Ref. 13.) In the case of F-actin filaments, the theoretically predicted power law dependence ${ }^{17,21-28} \sim t^{3 / 4}$ of the mean square displacement has been confirmed. ${ }^{14,15}$ With the progress in experimental techniques, in particular, in the field of FCS, there is a paramount interest to extract the full dynamic information from the measured signal and not only power laws. Naturally, this provides a more detailed insight into the physical properties of biological macromolecules. To extract the corresponding dynamical quantities, e.g., the segmental mean square displacement, an adequate theoretical description is required of both, the molecular dynamics as well as the FCS correlation function, ${ }^{14,15}$ where a polymer model independent approach is desirable. ${ }^{15}$

Theoretically, a semiflexible polymer is typically modeled as a differentiable space curves with the bending energy 


$$
V_{B}=\frac{\epsilon k_{B} T}{2} \int_{-L / 2}^{L / 2}\left(\frac{\partial^{2} \boldsymbol{r}(s)}{\partial s^{2}}\right)^{2} d s,
$$

where $L$ is the length of the polymer, $\boldsymbol{r}(s)$ the position in space of the point at $s(-L / 2 \leqslant s \leqslant L / 2)$ along the chain contour, $\epsilon$ the bending elastic constant which is related to the persistence length, $T$ the temperature, and $k_{B}$ the Boltzmann constant. An important feature of a linear polymer is its $\left(\right.$ nearly $\left.^{29}\right)$ fixed contour length. In mathematical terms, the constraint $\boldsymbol{u}(s)^{2}=1$, with $\boldsymbol{u}(s)=\partial \boldsymbol{r}(s) / \partial s$, has to be taken into account in the theoretical treatment. This model of a semiflexible polymer is referred to as Kratky-Porod wormlike chain. ${ }^{30}$ The adequate consideration of the constraint is a major challenge. Two strategies are utilized to arrive at a tractable model: (i) The constraint $\boldsymbol{u}(s)^{2}=1$ is relaxed and replaced by the condition $\left\langle\boldsymbol{u}(s)^{2}\right\rangle=1$, i.e., only the average of the square of the tangent vector has to be equal to unity. This leads to what may be called Gaussian model of a semiflexible polymer. ${ }^{10,31-40}$ There are a number of results confirming the usefulness of such an approach for structural ${ }^{10,41,42}$ as well as dynamical properties. ${ }^{9,14,16,17,20}$ (ii) To determine the structural properties of the Kratky-Porod wormlike chain, various approximation schemes have been applied ${ }^{4-47}$ and a number of equilibrium properties have been obtained successfully. ${ }^{5,74,48}$ The evaluation of the polymer dynamics requires other strategies. Usually the dynamical properties of the Kratky-Porod wormlike chain are evaluated in the limit of a weakly bending rod only, i.e., in the limit $L / l_{p} \ll 1 .^{22,25,26,49}$ Here, only the undulations transverse to the major axis of the rodlike polymer are taken into account assuming that the orientation and center-of-mass position are virtually fixed. As a major drawback, the rotational motion of the polymer is not captured in the treatment. This has no consequences on the dynamical properties as long as time scales are considered which are much smaller than the rotational relaxation time. However, the FCS correlation function is typically not only determined by the intramolecular motion (without rotation) but rather by the rotational motion and the center-of-mass diffusion. ${ }^{14}$ To what extent these individual parts contribute to the correlation function depends on various parameters such as polymer length in comparison to the size of the detection volume and the persistence length. Hence, for a quantitative analysis of the FCS correlation function all relaxation processes have to be taken into account.

In this article, the dynamics of semiflexible macromolecules and the FCS correlation function will be discussed for $L / l_{p}<1$. Particular attention will be paid to contributions of the rotational and center-of-mass dynamics. As will be shown, neglect of the rotational diffusion will change the FCS correlation function significantly for a certain range of polymer lengths. Moreover, an analytical approximation for the FCS correlation is presented, which agrees well with the full numerical determined function in the limit $L / l_{p} \ll 1$. This expression relates the polymer mean square displacement to the FCS correlation in a model independent way and reduces to that presented in Ref. 15 in the limit $L \rightarrow \infty$. The theoretical results will be compared with recent measurements of the FCS correlation function of actin filaments. ${ }^{15}$
The Gaussian semiflexible polymer model ${ }^{40}$ is used in the limit of a weakly bending rod. For a comprehensive description of the theoretical basis, the relevant equilibrium and dynamical properties of the model are described with specific account of the fact that only the bending modes transverse to the rod axis contribute the intramolecular relaxation. Our previous studies ${ }^{14,16}$ exploited the preaveraging approximation for the hydrodynamic interactions which is appropriate for flexible polymers. The anisotropy of rodlike polymers allows for another approximation of the Oseen tensor leading to quantitative differences for the diffusion coefficient and relaxation times. Compared to the first studies of the weakly bending rod dynamics, ${ }^{49}$ the Gaussian semiflexible model takes the rod rotational motion consistently into account. ${ }^{3}$ As will be shown, this leads to simpler and more adequate mode amplitude correlation functions than those following from the original approach. ${ }^{49-51}$

The article is organized as follows. In the next section the model is described and its equation of motion is solved. In Sec. III, the center-of-mass as well as the intramolecular mean square displacement is discussed and relations to previous results are established. The theoretical expressions for the FCS correlation function are derived in Sec. IV. Results for the mean square displacement and the FCS correlation function are presented in Sec. V. In particular, the theoretical results are compared with experimental measurements on actin filament. Finally, Sec. VI summarizes the findings.

\section{DYNAMICS OF SEMIFLEXIBLE POLYMERS}

The equilibrium properties of Gaussian semiflexible polymers have been described in a number of articles. ${ }^{10,20,39,40}$ Therefore, I will here summarize the aspects relevant for the analysis of the dynamics of weakly bending polymer only.

In brief, the semiflexible linear polymer is modeled as a continuous, differential space curve $\boldsymbol{r}(s)$. The chain connectivity is captured by the constraint $\left\langle(\partial \boldsymbol{r}(s) / \partial s)^{2}\right\rangle=1$ and bending restrictions lead to the potential $V_{B}$ of Eq. (1). Evaluation of the partition function yields in $\hat{d}$ dimensions,

$$
\begin{aligned}
Z= & \int \exp \left(-\nu \int_{-L / 2}^{L / 2}\left(\frac{\partial \boldsymbol{r}(s)}{\partial s}\right)^{2} d s-\frac{\boldsymbol{\epsilon}}{2} \int_{-L / 2}^{L / 2}\left(\frac{\partial^{2} \boldsymbol{r}(s)}{\partial s^{2}}\right)^{2} d s\right. \\
& \left.-\nu_{0}\left[\left(\frac{\partial \boldsymbol{r}(-L / 2)}{\partial s}\right)^{2}+\left(\frac{\partial \boldsymbol{r}(L / 2)}{\partial s}\right)^{2}\right]\right) \mathcal{D}^{d} x
\end{aligned}
$$

The Lagrangian multipliers $\nu, \nu_{0}$, and $\epsilon$ are given by

$$
\nu=\frac{\hat{d} p}{2}, \quad \nu_{0}=\frac{\hat{d}}{4}, \quad \epsilon=\frac{\hat{d}}{4 p}, \quad p=\frac{1}{2 l_{p}},
$$

(for $\hat{d}=3$, see Ref. 14), and $p$ is an abbreviation related to the persistence length $l_{p}$. The terms with the first derivative in $r$ capture the chain flexibility, i.e., they account for the chain entropy. The chain ends behave differently from the rest of the polymer, which is captured in the terms with $\nu_{0}$. The Gaussian model typically provides the same first and second moments as the Kratky-Porod wormlike chain model. ${ }^{40}$ Examples are the following: 
(i) the mean square end-to-end distance and the radius of gyration. In particular, the mean square distance among two points $s$ and $s^{\prime}$ is

$$
\begin{aligned}
\sigma\left(s-s^{\prime}\right) & =\left\langle\left(\boldsymbol{r}(s)-\boldsymbol{r}\left(s^{\prime}\right)\right)^{2}\right\rangle \\
& =\frac{\left|s-s^{\prime}\right|}{p}-\frac{1}{2 p^{2}}\left[1-\exp \left(-2 p\left|s-s^{\prime}\right|\right)\right],
\end{aligned}
$$

which gives $\sigma(s)=s^{2}$ in the limit $L / l_{p} \ll 1$;

(ii) the correlation function of tangent vectors,

$$
\left\langle\boldsymbol{u}(s) \boldsymbol{u}\left(s^{\prime}\right)\right\rangle=\exp \left(-2 p\left|s-s^{\prime}\right|\right) ;
$$

(iii) average contour length, ${ }^{49}$

$\langle L\rangle=\int_{-L / 2}^{L / 2}\langle\boldsymbol{u}(s) \boldsymbol{u}(s)\rangle d s=L ;$

(iv) projection of the end-to-end vector onto the tangent at the chain end,

$$
\langle\boldsymbol{r}(L) \boldsymbol{u}(L)\rangle=\frac{1}{2 p}[1-\exp (-2 p L)] .
$$

Higher order moments do not necessarily agree with those of the Kratky-Porod wormlike chain.

The equation of motion of the Gaussian semiflexible polymer, including hydrodynamic interactions in terms of the Ossen tensor, ${ }^{19,52}$ is given by the Langevin equation, ${ }^{14,17}$

$$
\begin{aligned}
\frac{\partial}{\partial t} \boldsymbol{r}(s, t)= & \int_{-L / 2}^{L / 2} \mathbf{H}\left(\boldsymbol{r}(s), \boldsymbol{r}\left(s^{\prime}\right)\right)\left[2 \nu k_{B} T \frac{\partial^{2}}{\partial s^{\prime 2}} \boldsymbol{r}\left(s^{\prime}, t\right)\right. \\
& \left.-\epsilon k_{B} T \frac{\partial^{4}}{\partial s^{\prime 4}} \boldsymbol{r}\left(s^{\prime}, t\right)+\boldsymbol{\Gamma}\left(s^{\prime}, t\right)\right] d s^{\prime},
\end{aligned}
$$

with the boundary conditions

$$
\begin{aligned}
& {\left[2 \nu \frac{\partial}{\partial s} \boldsymbol{r}(s, t)-\epsilon \frac{\partial^{3}}{\partial s^{3}} \boldsymbol{r}(s, t)\right]_{ \pm L / 2}=0,} \\
& {\left[2 \nu_{0} \frac{\partial}{\partial s} \boldsymbol{r}(s, t) \pm \epsilon \frac{\partial^{2}}{\partial s^{2}} \boldsymbol{r}(s, t)\right]_{ \pm L / 2}=0 .}
\end{aligned}
$$

Note, the boundary conditions are independent of the dimension $\hat{d}$. The stochastic force $\boldsymbol{\Gamma}(s, t)$ is assumed to be stationary, Markovian, and Gaussian with zero mean. ${ }^{19,53}$ The hydrodynamic tensor is defined as $\mathbf{H}\left(\boldsymbol{r}(s), \boldsymbol{r}\left(s^{\prime}\right)\right)=\mathbf{Q}(\boldsymbol{r}(s)$ $\left.\boldsymbol{- r}\left(s^{\prime}\right)\right)+\mathbf{I} \delta\left(s-s^{\prime}\right) / 3 \pi \eta$, where $\mathbf{Q}\left(\boldsymbol{r}(s)-\boldsymbol{r}\left(s^{\prime}\right)\right)$ is the Oseen tensor.

In order to transform the nonlinear equation (8) into a linear equation, the following presumption can be applied: The hydrodynamic tensor is replaced by its spacial isotropic averaged expression according to Zimm, ${ }^{18,19}$ or the hydrodynamic tensor of a rodlike object is utilized. To elucidate the quantitative differences among the two assumptions, both possibilities will be described.

Preaveraging approximation. Using the Gaussian joint probability distribution, ${ }^{14}$ the position dependent hydrodynamic tensor turns into an isotropic tensor which depends on the contour coordinates only, i.e., $\mathbf{H}\left(s, s^{\prime}\right)=\mathbf{I}\left[\delta\left(s-s^{\prime}\right) / 3 \pi \eta+Q\left(s-s^{\prime}\right)\right]$, where the term with the delta function accounts for the local friction, $\eta$ is the solvent viscosity, and

$Q(s)=\frac{\Theta(|s|-d)}{3 \pi \eta} \sqrt{\frac{3}{2 \pi \sigma(s)}} \exp \left(-\frac{3 d^{2}}{2 \sigma(s)}\right)$.

Here, the Heaviside step function $\Theta$ is introduced to exclude self-interactions and $d$ is the thickness of the molecule. $^{14,24}$

(ii) Rodlike polymer. For an infinitely thin rod, the difference between two points along the contour is $\boldsymbol{r}(s)$ $\boldsymbol{- r}\left(s^{\prime}\right)=\left(s-s^{\prime}\right) \boldsymbol{u}$, with the unit vector $\boldsymbol{u}$ pointing in the direction of the long rod axis. By partitioning the Oseen tensor in components parallel and perpendicular to the rod axis, $\mathbf{Q}\left(\boldsymbol{r}(s)-\boldsymbol{r}\left(s^{\prime}\right)\right)=Q_{\|} \boldsymbol{u}+Q_{\perp} \boldsymbol{v}$, where $\boldsymbol{v}$ is a unit vector perpendicular to $\boldsymbol{u}$, we arrive at

$$
Q_{\perp}(s)=Q_{\|}(s) / 2=\frac{\Theta(|s|-d)}{8 \pi \eta|s|} .
$$

To solve the equation of motion with either Eq. (11) or (12), an eigenfunction expansion in terms of the eigenfunctions of the eigenvalue equation

$$
\epsilon k_{B} T \frac{d^{4}}{d s^{4}} \psi_{n}(s)-2 \nu k_{B} T \frac{d^{2}}{d s^{2}} \psi_{n}(s)=\xi_{n} \psi_{n}(s)
$$

is used. The eigenfunctions are given by

$$
\begin{aligned}
& \psi_{0}=\sqrt{\frac{1}{L}}, \\
& \psi_{n}(s)=\sqrt{\frac{c_{n}}{L}}\left(\zeta_{n}^{\prime} \frac{\sinh \zeta_{n}^{\prime} s}{\cosh \zeta_{n}^{\prime} L / 2}+\zeta_{n} \frac{\sin \zeta_{n} s}{\cos \zeta_{n} L / 2}\right), \quad \forall n \text { odd }, \\
& \psi_{n}(s)=\sqrt{\frac{c_{n}}{L}}\left(\zeta_{n}^{\prime} \frac{\cosh \zeta_{n}^{\prime} s}{\sinh \zeta_{n}^{\prime} L / 2}-\zeta_{n} \frac{\cos \zeta_{n} s}{\sin \zeta_{n} L / 2}\right), \quad \forall n \text { even. }
\end{aligned}
$$

The $c_{n}$ 's follow from the normalization condition. The wave numbers $\zeta_{n}$ and $\zeta_{n}^{\prime}$, where $\zeta_{n}^{\prime 2}-\zeta_{n}^{2}=4 p^{2}$, are determined by the boundary conditions (9) and (10) and are related to the eigenvalues via $\xi_{n}=k_{B} T\left(\epsilon \zeta_{n}^{4}+2 \nu \zeta_{n}^{2}\right) . \psi_{0}$ describes the translational motion of the whole molecule.

In the limit $p L<1$, the eigenfunctions and eigenvalues $(n>1)$ agree with those of the weakly bending rod model ${ }^{49}$ for $\hat{d}=2$. However, it is important to keep in mind that the first mode of the above model is finite for $0<p L<1$ and hence is different from the pure rotational mode of the Aragón and Pecora model ${ }^{49}$ (cf. Fig. 1 of Ref. 3). As we have shown in Ref. 3, the first mode corresponds to the rotational motion of the rodlike object in the limit $p L \rightarrow 0$. Hence, the Gaussian semiflexible polymer model not only captures the bending modes but also the rotation of a rodlike object. This will be important for the further discussion of the polymer dynamics.

In the limit $p L \lesssim 0.1$, the eigenfunctions read explicitly as 


$$
\begin{aligned}
& \psi_{n}(s)=\sqrt{\frac{1}{L}}\left(\frac{\sinh \zeta_{n} s}{\sinh \zeta_{n} L / 2}+\frac{\sin \zeta_{n} s}{\sin \zeta_{n} L / 2}\right), \quad n>1, \text { odd }, \\
& \psi_{n}(s)=\sqrt{\frac{1}{L}}\left(\frac{\cosh \zeta_{n} s}{\cos \zeta_{n} L / 2}+\frac{\cos \zeta_{n} s}{\cos \zeta_{n} L / 2}\right), \quad n>1, \text { even. }
\end{aligned}
$$

With $\zeta_{n}=(2 n-1) \pi / 2 L(n>1)$, the corresponding eigenvalues are

$$
\xi_{n}=\frac{\epsilon \pi^{4}(2 n-1)^{4} k_{B} T}{16 L^{4}},
$$

i.e., the well-known cubic dependence is obtained. ${ }^{3,49}$ In the limit $p L \rightarrow 0$ the eigenfunction of the first mode is given by

$$
\psi_{1}(s)=\sqrt{\frac{12}{L^{3}}} s,
$$

with the eigenvalue

$$
\xi_{1}=\frac{48 \nu_{0} k_{B} T}{L^{3}},
$$

which is independent of the persistence length.

The solution of Eq. (8) is then obtained by the eigenfunction expansions

$$
\boldsymbol{r}(s, t)=\sum_{n=0}^{\infty} \boldsymbol{\chi}_{n}(t) \psi_{n}(s), \quad \boldsymbol{\Gamma}(s, t)=\sum_{n=0}^{\infty} \boldsymbol{\Gamma}_{n}(t) \psi_{n}(s),
$$

which yields

$$
\frac{\partial}{\partial t} \boldsymbol{\chi}_{n}(t)=\sum_{m=0}^{\infty}\left(Q_{n m}+\frac{\delta_{n m}}{3 \pi \eta}\right)\left(-\frac{3 \pi \eta}{\tau_{m}} \boldsymbol{\chi}_{m}(t)+\boldsymbol{\Gamma}_{m}(t)\right) .
$$

The $\tau_{n}$ 's are the relaxation times in the free draining limit and are related to the eigenvalues $\xi_{n}$ via $\tau_{n}=\gamma / \xi_{n}$, where $\gamma$ $=3 \pi \eta$ is the friction constant per length. The $Q_{n m}$ 's are the matrix elements of either the preaveraged Oseen tensor $Q\left(s-s^{\prime}\right)$ [Eq. (11)] or $Q_{\perp}\left(s-s^{\prime}\right)$ [Eq. (12)]—depending on the applied approximation-in terms of the eigenfunctions $\psi_{n}(s)$. A numerical calculation shows that the matrix $Q_{n m}$ is almost diagonal over the whole range of the flexibility parameter $p L{ }^{54}$ Hence, $Q_{n m} \approx \delta_{n m} Q_{n n}$ and the stationary state solution for $\boldsymbol{\chi}_{n}(t)$ is easily obtained as

$$
\chi_{n}(t)=\frac{\tau_{n}}{3 \pi \eta \widetilde{\tau}_{n}} \int_{-\infty}^{t} e^{-\left(t-t^{\prime}\right) \tilde{\tau}_{n}} \boldsymbol{\Gamma}_{n}\left(t^{\prime}\right) d t^{\prime}
$$

with the relaxation times

$$
\tilde{\tau}_{n}=\frac{\tau_{n}}{1+3 \pi \eta Q_{n n}} .
$$

The time correlation functions of appropriate polymer properties are often required when the above results are applied to experimental measurements. Many of these correla- tion functions can simply be obtained from the correlation functions of the amplitudes $\boldsymbol{\chi}_{n}$. A straightforward calculation yields

$$
\left\langle\boldsymbol{\chi}_{n}(t) \boldsymbol{\chi}_{m}(0)\right\rangle=\frac{k_{B} T}{\pi \eta} \tau_{n} \delta_{n m} e^{-t t \tilde{\tau}_{n}}, \quad n, m>0 .
$$

Evidently, the correlation function can be reformulated as $\left\langle\boldsymbol{\chi}_{n}(t) \boldsymbol{\chi}_{m}(0)\right\rangle=\left\langle\boldsymbol{\chi}_{n}(0) \boldsymbol{\chi}_{m}(0)\right\rangle e^{-t / \tilde{\tau}_{n}}, \quad$ with $\quad\left\langle\boldsymbol{\chi}_{n}(0) \boldsymbol{\chi}_{m}(0)\right\rangle$ $=k_{B} T \tau_{n} \delta_{n m} / \pi \eta$. This seemingly simple interrelation has important consequences for the further analysis of the weakly bending rod dynamics. First of all, the extension of the weakly bending rod equations of motion ${ }^{49}$ by the term containing $\nu$ [cf. Eq. (8)] leads to much simpler normal mode amplitude correlation functions than those obtained by Aragón. ${ }^{49-51}$ In particular, the various modes form an orthogonal set. The reason is the more adequate treatment of the first mode, which is artificially introduced in the weakly bending rod model. ${ }^{49}$ Note, in the limit of a flexible polymer, the weakly bending rod model is not applicable. Secondly, $\left\langle\boldsymbol{\chi}_{n}(0) \boldsymbol{\chi}_{m}(0)\right\rangle$ is an equilibrium average and is not at all related to the polymer dynamics. There are various ways to determine the equilibrium average. ${ }^{49}$ For the considered Gaussian model, the eigenfunction expansion [Eq. (20)] diagonalizes the exponent in the partition function [Eq. (2)]. The average is then easily calculated as $\left\langle\boldsymbol{\chi}_{n}(0) \boldsymbol{\chi}_{m}(0)\right\rangle$ $=3 k_{B} T \delta_{n m} / \xi_{n}$, in agreement with Eq. (24). Note, since this is an equilibrium average, the Lagrangian multipliers [Eq. (3)] with $\hat{d}=3$ have to be used in the expression for $\xi_{n}$ [Eq. (17)]. For $p L \ll 1$, the correlation functions for modes with $n>1$ are then given by

$$
\left\langle\boldsymbol{\chi}_{n}(0)^{2}\right\rangle=\frac{64 p L^{4}}{\pi^{4}(2 n-1)^{4}} .
$$

This relation will turn out to be useful in the calculation of the mean square displacement of the polymer.

\section{MEAN SQUARE DISPLACEMENT}

The mean square displacement of the point $s$ is given by

$$
\begin{aligned}
\left\langle\Delta \boldsymbol{r}(s, t)^{2}\right\rangle & =\left\langle[\boldsymbol{r}(s, t)-\boldsymbol{r}(s, 0)]^{2}\right\rangle \\
& =\left\langle\Delta \boldsymbol{r}_{\mathrm{cm}}(t)^{2}\right\rangle+6 k_{B} T \sum_{n=1}^{\infty} \xi_{n}^{1} \psi_{n}(s)^{2}\left(1-e^{-t / \tilde{\tau}_{n}}\right) .
\end{aligned}
$$

The segmental mean square displacement consist of two parts, a contribution from the center-of-mass motion $\left\langle\Delta \boldsymbol{r}_{\mathrm{cm}}(t)^{2}\right\rangle$ and a term capturing the intramolecular dynamics. Later on, the mean square displacement averaged over the polymer contour $\left(\left\langle\Delta \boldsymbol{r}(t)^{2}\right\rangle=\int\left\langle\Delta \boldsymbol{r}(s, t)^{2}\right\rangle d s / L\right)$ will be used, which reads

$$
\left\langle\Delta \boldsymbol{r}(t)^{2}\right\rangle=\left\langle\Delta \boldsymbol{r}_{\mathrm{cm}}(t)^{2}\right\rangle+\frac{6 k_{B} T}{L} \sum_{n=1}^{\infty} \xi_{n}^{-1}\left(1-e^{-t / \tilde{\tau}_{n}}\right) .
$$




\section{A. Center-of-mass mean square displacement}

The center-of-mass mean square displacement of a rodlike object is related to the diffusion coefficient $D$ via

$$
\left\langle\Delta \boldsymbol{r}_{\mathrm{cm}}(t)^{2}\right\rangle=6 D t=\left(4 D_{\perp}+2 D_{\|}\right) t,
$$

where $D_{\|}$and $D_{\perp}$ denote the diffusion coefficients parallel and perpendicular to the rod axis. ${ }^{19,52}$ From the equation of motion for $\boldsymbol{r}_{\mathrm{cm}}$, the expression

$$
D_{\perp}=\frac{k_{B} T}{3 \pi \eta L}+\frac{k_{B} T}{L^{2}} \int_{-L / 2}^{L / 2} \int_{-L / 2}^{L / 2} Q_{\perp}\left(s-s^{\prime}\right) d s d s^{\prime}
$$

is obtained, when the Oseen tensor components of Eq. (12) are used. Analogously, the coefficient $D_{\|}$follows when $Q_{\perp}$ is replaced by $Q_{\|}$. Evaluation of the integrals yields

$$
D_{\perp}=D_{\|} / 2=\frac{k_{B} T}{4 \pi \eta L} \ln (L / d),
$$

for $L / d \gg 1$. (Note, a more precise hydrodynamic calculation for a cylinder gives corrections. ${ }^{19,52,55}$ ) The diffusion coefficient then reads

$$
D=\frac{k_{B} T}{3 \pi \eta L} \ln (L / d) \text {. }
$$

(The same expression is obtained, if the spacial averaged Oseen tensor $\mathbf{Q}=\mathbf{I} \Theta(|s|-d) / 6 \pi \eta|s|$ is used right from the start.) Since $D_{\|}$is twice as large as $D_{\perp}$, both components have to be taken into account in the analysis of the large scale dynamics of rodlike polymers. It is not possible to focus on the transverse diffusion only. ${ }^{15}$ As soon as the centerof-mass diffusion is relevant for the overall rod motion, both components are equally important.

Within the preaveraging approximation, the same type of calculation yields

$$
D_{p}=\sqrt{\frac{6}{\pi}} \frac{k_{B} T}{3 \pi \eta L} \ln (L / d)=\sqrt{\frac{6}{\pi}} D,
$$

i.e., $D_{p}$ is about $40 \%$ larger than the diffusion coefficient of a rod.

Since the approximation used to derive Eq. (12) applies in the rod limit only and the preaveraging approximation is suitable for flexible polymers, there is a crossover from $D$ to $D_{p}$ for certain persistence lengths. It remains to be shown at which $l_{p}$ this crossover occurs.

\section{B. Intramolecular mean square displacement}

Equilibrium properties at all stiffnesses as well as dynamical properties for flexible polymers ${ }^{14,16}$ are calculated in three dimensional space, i.e, $\hat{d}=3$ is used in Eq. (3). In the limit of a weakly bending rod, fluctuations along the rod axis are suppressed, because of the inextensibility constraint, and the dynamics is essentially two dimensional-transverse to the main rod axis. ${ }^{49}$ Thus, the Lagrangian multipliers [Eq. (3)] with $\hat{d}=2$ have to be used for the calculation of the relaxation times $\widetilde{\tau}_{n}$, and the parameter $\epsilon=1 / 2 p$ corresponds to that of the Kratky-Porod model. ${ }^{40}$ The relaxation times $\widetilde{\tau}_{n}$ are then given by

$$
\widetilde{\tau}_{n}=\frac{96 \eta p L^{4}}{\pi^{3} k_{B} T(2 n-1)^{4}} \frac{1}{1+3 \pi \eta Q_{n n}^{\perp}},
$$

for $p L<1$ and $n>1$, and the Oseen tensor transverse to the rod axis [Eq. (12)]. For large mode numbers $n$ and for $d / L$ $\ll 1$, the matrix elements $Q_{n n}^{\perp}$ are approximately given by

$$
Q_{n n}^{\perp}=\frac{1}{4 \pi \eta} \int_{d}^{L} \frac{\cos \zeta_{n} s}{s} d s=-\frac{C+\ln \left(\zeta_{n} d\right)}{4 \pi \eta},
$$

where $C=0.5772 \cdots$ is Euler's constant, which leads to

$$
\widetilde{\tau}_{n}=\frac{8 \eta p L^{4}}{\pi^{3} k_{B} T n^{4} \ln (L / n \pi d)} .
$$

These relaxation times agree with those of Refs. 25 and 26 for $p=1 / 2 l_{p}{ }^{15}$

In the preaveraging approximation, the relaxation times $\widetilde{\tau}_{n}^{p}=\widetilde{\tau}_{n} \sqrt{3 \pi / 32}$ are obtained by the same procedure. Hence, preaveraging underestimates the relaxation times by almost a factor of 2.

The rotational relaxation time follows from the correlation function $\langle\boldsymbol{u}(t) \boldsymbol{u}(0)\rangle .{ }^{19,52}$ In the limit $p L \rightarrow 0$, only the first mode contributes to the relaxation, ${ }^{17}$ which yields, with the eigenfunction [Eq. (18)] and the equilibrium correlation function $\left\langle\boldsymbol{\chi}_{1}^{2}\right\rangle$,

$$
\langle\boldsymbol{u}(t) \boldsymbol{u}(0)\rangle=e^{-t / \widetilde{\tau}_{1}} .
$$

Hence, the rotational relaxation time is given by $\tau_{r}=\widetilde{\tau}_{1}$ and the rotational diffusion coefficient is $D_{r}=1 / 2 \widetilde{\tau}_{1}$. ${ }^{19}$ Similar to the calculations for $n>1$, the relaxation time itself reads

$$
\widetilde{\tau}_{1}=\frac{\pi \eta L^{3}}{6 k_{B} T \ln (L / d)}
$$

for $L / d \gg 1$ and $\epsilon=1 / 2 p$. This relaxation time is, to leading order, identical with the relaxation time of a rigid rod. ${ }^{1,52,55}$

Putting all pieces together, the following averaged segmental mean square displacement (27) is obtained:

$$
\begin{aligned}
\left\langle\Delta \boldsymbol{r}(t)^{2}\right\rangle= & 6 D t+\frac{L^{2}}{6}\left(1-e^{-t / \tilde{\tau}_{1}}\right) \\
& +\frac{8 p L^{3}}{\pi^{4}} \sum_{n=2}^{\infty} \frac{1}{(n-1 / 2)^{4}}\left(1-e^{-t / \tilde{\tau}_{n}}\right),
\end{aligned}
$$

in the limit $p L \ll 1$ and $L / d \gg 1$. Apart from the factor $1 / 2$ for the mode numbers, the sum over modes in Eq. (38) agrees with the mean square displacement provided in Ref. 15. The latter total mean square displacement applies to the dynamics transverse to the rod axis only, whereas Eqs. (26) and (38) yield the segmental mean square displacement averaged over all rod orientations. In particular, no extra consideration of the mean square displacement parallel to the rod axis is necessary for the provided approach. More importantly, Eqs. (26) and (38) capture the rotational motion of the semiflexible polymer.

For $t / \widetilde{\tau}_{2} \ll 1$, a large number of modes contribute to the mean square displacement. Converting the sum of modes into an integral, Eq. (38) reads 


$$
\begin{aligned}
\left\langle\Delta \boldsymbol{r}(t)^{2}\right\rangle= & 6 D t+\frac{L^{2}}{6}\left(1-e^{-t / \widetilde{\tau}_{1}}\right) \\
& +0.27\left[\frac{k_{B} T p^{1 / 3} \ln (L / \pi d)}{\eta} t\right]^{3 / 4},
\end{aligned}
$$

for $\eta p d^{4} / k_{B} T \ll t \ll \eta p L^{4} / k_{B} T$. As expected, the well-known $t^{3 / 4}$ dependence of the intramolecular mean square displacement is reproduced. ${ }^{17,21-26}$ The logarithm captures the hydrodynamic interactions, where $\ln (L / n \pi d) \approx \ln (L / \pi d)$ [Eq. (35)] has been used. By an iteration procedure, the dependence of $\widetilde{\tau}_{n}$ [Eq. (35)] on the mode number can be taken into account. A first iteration step turns the $t^{3 / 4}$ term of Eq. (39) into $0.097\left(k_{B} T p^{1 / 3} t \ln \left[k_{B} T t \ln (L / \pi d) / 8 \pi \eta p d^{4}\right] / \eta\right)^{3 / 4}$ in accordance with the expression derived in Ref. $25 .{ }^{15}$

\section{FCS CORRELATION FUNCTION}

FCS (Refs. 11 and 56-63) is a powerful experimental technique to study the diffusional motion and intramolecular dynamics of fluorescently labeled molecules at nanomolar concentration. ${ }^{16}$ In FCS, the fluctuations of the fluorescent light intensity $I(t)=\langle I\rangle+\delta I(t)$ are detected via the correlation function,

$$
g(t)=\frac{\left\langle\delta I\left(t^{\prime}\right) \delta I\left(t^{\prime}+t\right)\right\rangle}{\langle I\rangle^{2}} .
$$

In the absence of additional photophysical processes and chemical reactions, the fluorescence signal fluctuates due to the Brownian motion of the labeled particles through a femtoliter-size volume whose shape can be approximated by a three dimensional Gaussian, ${ }^{64}$

$$
W(\boldsymbol{r})=W_{0} \exp \left(-2 \frac{x^{2}+y^{2}}{r_{0}^{2}}\right) \exp \left(-2 \frac{z^{2}}{z_{0}^{2}}\right),
$$

with the lateral and axial extensions $r_{0}$ and $z_{0}$, respectively. Adopting the approach of Ref. 65, the FCS correlation can then be written as

$$
g(t)=\frac{1}{\bar{c} V_{\mathrm{eff}}} \frac{\int \Omega(\boldsymbol{q}) S(\boldsymbol{q}, t) d^{3} q}{\int \Omega(\boldsymbol{q}) d^{3} q},
$$

where $\Omega(\boldsymbol{q})=\mathrm{FT}\{W(\boldsymbol{r})\}_{\boldsymbol{q}} \mathrm{FT}\{W(\boldsymbol{r})\}_{-\boldsymbol{q}}$ is the filter function in Fourier space and $S(\boldsymbol{q}, t)$ is the dynamic structure factor. $\bar{c}$ is the mean particle concentration and $V_{\text {eff }}=\pi^{3 / 2} r_{0}^{2} z_{0}^{2}$ the socalled effective volume.

For the theoretical study, it is convenient to normalize the correlation function in such a way that it is unity at $t$ $=0$. Hence, the correlation function

$$
G(t)=\frac{\int \Omega(\boldsymbol{q}) S(\boldsymbol{q}, t) d^{3} q}{\int \Omega(\boldsymbol{q}) S(\boldsymbol{q}) d^{3} q},
$$

with $S(\boldsymbol{q})=S(\boldsymbol{q}, 0)$, will be considered in the following.

In Sec. II, a continuum description of a rodlike polymer is adopted. Still, a polymer with a finite number of labels can be described. The dynamic structure factor appearing in the FCS correlation function [Eq. (43)] has just to be evaluated taking only the labeled parts of the polymer into account. ${ }^{14}$ Here, we limit the discussion to a fully labeled polymer, i.e., a polymer with a continuous distribution of labels. The dynamic structure factor is then given by ${ }^{14,19}$

$$
S(\boldsymbol{q}, t)=\frac{1}{L^{2}} \int_{-L / 2}^{L / 2} \int_{-L / 2}^{L / 2}\left\langle\exp \left(-i \boldsymbol{q}\left[\boldsymbol{r}(s, t)-\boldsymbol{r}\left(s^{\prime}, 0\right)\right]\right)\right\rangle d s d s^{\prime},
$$

where $\boldsymbol{q}$ is the scattering vector. Since the distribution of distance is Gaussian in the applied model (cf. Sec. II), the dynamic structure factor is easily calculated as ${ }^{14,19}$

$$
S(\boldsymbol{q}, t)=\frac{1}{L^{2}} \int_{-L / 2}^{L / 2} \int_{-L / 2}^{L / 2} \exp \left(-\frac{q^{2}}{6} \Phi\left(s, s^{\prime}, t\right)\right) d s d s^{\prime},
$$

where

$$
\begin{aligned}
\Phi\left(s, s^{\prime}, t\right)= & \left\langle\left[\boldsymbol{r}(s, t)-\boldsymbol{r}\left(s^{\prime}, 0\right)\right]^{2}\right\rangle \\
= & 6 D t+\sigma\left(s-s^{\prime}\right) \\
& +6 k_{B} T \sum_{n=1}^{\infty} \xi_{n}^{-1} \psi_{n}(s) \psi_{n}\left(s^{\prime}\right)\left(1-e^{-t / \widetilde{\tau}_{n}}\right),
\end{aligned}
$$

and $\sigma$ is defined in Eq. (4). (Note, similar to the mean square displacements (26) and (38), $\xi_{n}$ has to be determined with $\hat{d}=3$ and $\tilde{\tau}_{n}$ with $\hat{d}=2$.) Integration over the $\boldsymbol{q}$ vector in Eq. (43) yields then the expression ${ }^{14}$

$$
\begin{aligned}
G(t)= & g_{0}^{-1} \int_{-L / 2}^{L / 2} \int_{-L / 2}^{L / 2}\left(1+\frac{2 \Phi\left(s, s^{\prime}, t\right)}{3 r_{0}^{2}}\right)^{-1} \\
& \times\left(1+\frac{2 \Phi\left(s, s^{\prime}, t\right)}{3 z_{0}^{2}}\right)^{-1 / 2} d s d s^{\prime}
\end{aligned}
$$

with

$$
g_{0}^{-1}=2 \int_{0}^{L}(L-s)\left(1+\frac{2 \sigma(s)}{3 r_{0}^{2}}\right)^{-1}\left(1+\frac{2 \sigma(s)}{3 z_{0}^{2}}\right)^{-1 / 2} d s .
$$

For large chain lengths $L \gg r_{0}$, the double integral is dominated by contributions with $s \approx s^{\prime}$ and hence is well approximated by a single integral. ${ }^{14,17,19}$ The expression $\Phi\left(s, s^{\prime}, t\right)$ is then given by $\Phi\left(s, s^{\prime}, t\right)=\Phi\left(s-s^{\prime}, t\right)=\sigma\left(s-s^{\prime}\right)$ $+\left\langle\Delta \boldsymbol{r}(s, t)^{2}\right\rangle$, with the mean square displacement Eq. (26). Replacing $\left\langle\Delta \boldsymbol{r}(s, t)^{2}\right\rangle$ by its average over the polymer contour [Eq. (27)], the following analytical expression for the FCS correlation function is obtained for a rodlike polymer $\left[\sigma(s) \approx s^{2}\right]:$

$$
\begin{aligned}
G(t)= & \frac{2 L f g_{0}^{-1}}{\sqrt{h_{1}}} \sqrt{\frac{3 r_{0}^{2}}{2\left(f^{2}-1\right)}} \arctan \left(\sqrt{\frac{2 L^{2}\left(f^{2}-1\right)}{3 r_{0}^{2} h_{1} h_{3}}}\right) \\
& -\frac{3 r_{0}^{2} f g_{0}^{-1}}{2 \sqrt{f^{2}-1}} \ln \left[\frac{\sqrt{f^{2}-1}-\sqrt{h_{3}}}{\sqrt{f^{2}-1}+\sqrt{h_{3}}} \frac{\sqrt{f^{2}-1}+\sqrt{h_{2}}}{\sqrt{f^{2}-1}-\sqrt{h_{2}}}\right],
\end{aligned}
$$

with the abbreviations

$$
h_{1}=1+\frac{2\left\langle\Delta \boldsymbol{r}(t)^{2}\right\rangle}{3 r_{0}^{2}},
$$




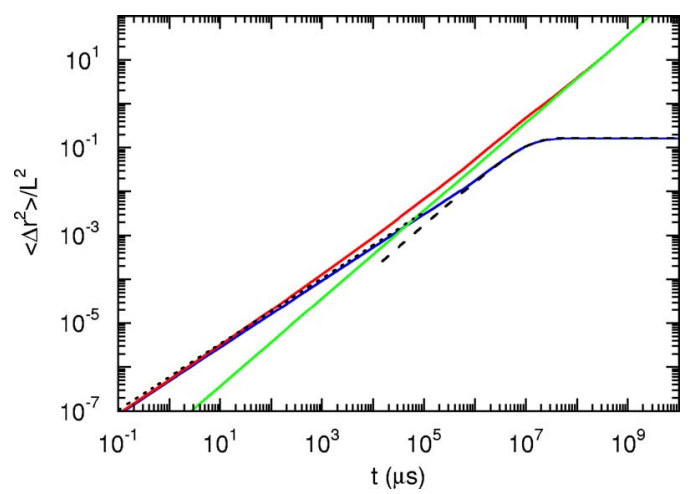

FIG. 1. (Color online) Mean square displacement (MSD) of a semiflexible polymer $(p L=0.1)$ according to Eq. (27). The top line is the total MSD, the bottom line the center-of-mass MSD, and the curve reaching a plateau the intramolecular MSD. The dotted line is the intramolecular part of the analytical expression (39) (last term). The dashed line is the MSD of the rotational motion of a rod [Eq. (53)].

$$
\begin{aligned}
& h_{2}=f^{2}+\frac{2\left\langle\Delta \boldsymbol{r}(t)^{2}\right\rangle}{3 r_{0}^{2}}, \\
& h_{3}=f^{2}+\frac{2 L^{2}}{3 r_{0}^{2}}+\frac{2\left\langle\Delta \boldsymbol{r}(t)^{2}\right\rangle}{3 r_{0}^{2}},
\end{aligned}
$$

and $f=z_{0} / r_{0}$. A similar, but more specific, expression has been derived in Ref. 14 [Eq. (50)], because an approximation has been used for the mean square displacement. The equation for $g_{0}^{-1}$ [Eq. (48)] illustrates that the transformation from a double to a single integral ${ }^{66}$ yields a term $(L-S)$. The logarithmic term in Eq. (49) originates from the $s$ part of that term. In Ref. 14, this contribution has been neglected.

No particular model for the polymer dynamics has been used to derive Eq. (49). Hence, by comparing the correlation function with experimental results, the averaged segmental mean square displacement can be determine in a model independent manner.

In Ref. 15, a different strategy is followed for the calculation of the FCS correlation function. The segmental displacement is split into components parallel and perpendicular to the main rod axis. Then, the ensemble average of $\left\langle e^{-i \boldsymbol{q}[\boldsymbol{r}(s, t)-\boldsymbol{r}(s, 0)]}\right\rangle$ is calculated for a fix $\boldsymbol{q}$. Finally, $G$ is averaged over all possible rod orientations and $\boldsymbol{q}$ 's. This approach neglects the rotational diffusion of the rod and thus applies at short times only. Strictly speaking, only the intramolecular dynamics is correctly described, since all dynamical quantities on the time scale of the rotational relaxation time, which is the longest intramolecular relaxation time, lack the contribution of rotational diffusion. Nevertheless, a FCS correlation function similar to Eq. (49) is derived. Both expression formally agree in the limit $L \rightarrow \infty$ and by neglecting the logarithmic term in Eq. (49). Still, the mean square displacements differ by the rotational motion. In the current study, the rotational motion is taken into account and, thus, a separation of the dynamics parallel and perpendicular to the rod axis is not possible. Nevertheless, in both approaches the weakly bending rod dynamics transverse to the rod axis [Eq. (38)] is taken into account.

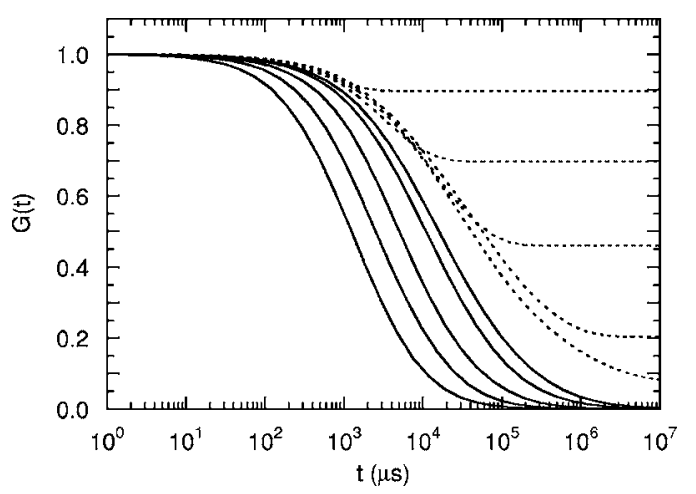

FIG. 2. FCS correlation functions for semiflexible polymers of various lengths: $L=0.5,1,2,5$, and $10 \mu \mathrm{m}$. The solid lines are determined including all modes (polymer length increases from left to right), whereas for the dotted lines the center-of-mass motion is omitted (length increases from top to bottom).

For rods of finite length, the arctan term is not contributing to the decay of the correlation function as long as $\left\langle\Delta \boldsymbol{r}(t)^{2}\right\rangle \ll L^{2}+3 f^{2} r_{0}^{2} / 2$. Thus, the expression of Ref. 15 is only applicable as long as the root mean square displacement is smaller than the length of the semiflexible polymer. Naturally, the correlation function of Ref. 15 is model independent in the same spirit as expression (49).

As is well known, the translational and rotational diffusions are coupled in the dynamic structure factor of a rod. ${ }^{52,67-71}$ Since the basis of the current analysis is a linear Langevin equation, this coupling is lost. The question, how severely the dynamic structure factor of the semiflexible polymer-and hence the FCS correlation function-is affected by this approximation remains to be clarified. However, I would expect that the coupling is less relevant for a semiflexible polymer than for a rigid rod.

\section{RESULTS AND DISCUSSION}

The analysis of the intramolecular dynamics requires rod lengths $L>r_{0}{ }^{14}$ For polymers with a radius of gyration $\left\langle\boldsymbol{r}_{g}^{2}\right\rangle^{1 / 2} \ll r_{0}$, the overall translational motion only is measured. Hence, polymers which satisfy the criteria $l_{p}>L>r_{0}$ will be considered in the following.

If not otherwise stated, the full numerical solution of the eigenvalue equation (13) is used to calculate dynamical quantities, i.e., the eigenfunctions [Eq. (14)] and eigenvalues with the wave numbers determined by the boundary conditions (9) and (10). Moreover, the matrix elements $Q_{n n}^{\perp}$ for the relaxation times $\tilde{\tau}_{n}$ [Eq. (23)] are numerically calculated with the same exact eigenfunctions.

\section{A. Mean square displacement}

To demonstrate the usefulness of the approximations derived in Sec. III, an example for the mean square displacement [Eq. (27)] is provided in Fig. 1. For the polymer the following parameters are used: $L=10 \mu \mathrm{m}, l_{p}=50 \mu \mathrm{m}$, and $L / d=10^{6}$. This yields $p L=0.1$, which implies that the polymer is rodlike. ${ }^{17}$ For the thickness a rather small value is used to reduce the influence of finite size correction terms. 
The parameters for the optical trap are $r_{0}=0.2 \mu \mathrm{m}$ and $f=5$. The temperature is $T=300 \mathrm{~K}$ and the viscosity $\eta$ $=10^{-3} \mathrm{~N} \mathrm{~s} / \mathrm{m}^{2}$.

Figure 1 shows that the bending modes determine the mean square displacement for $t \leqslant 10^{2} \mu \mathrm{s}$. For larger times the center-of-mass mean square displacement and the rotational motion contribute to $\left\langle\Delta \boldsymbol{r}^{2}\right\rangle$. The intramolecular mean square displacement saturates for times larger than the rotational relaxation time $t \geq 0.1 \mathrm{~s}$. The dashed line shows that in this time range the intramolecular mean square displacement is well described by the rod rotational motion, ${ }^{19,55}$

$$
\begin{aligned}
& \left\langle\Delta r_{r r}^{2}\right\rangle=\frac{L^{2}}{6}\left(1-e^{\left.-t / \tau_{r r}\right),}\right. \\
& \tau_{r r}=\frac{\pi \eta L^{3}}{6 k_{B} T[\ln (L / d)-0.66]} .
\end{aligned}
$$

The dotted line is the analytical approximation [Eq. (39)] (term with the logarithm only) for the weakly bending rod dynamics. This expression evidently captures the time dependence almost quantitatively. The slight shift to smaller times reduces for larger persistence length and larger $L / d$ ratios.

\section{B. FCS correlation function}

The dependence of the FCS correlation function of semiflexible polymers on chain length has been discussed in detail in Ref. 14. In that article, the relaxation times $\widetilde{\tau}_{n}$ are calculated with $\hat{d}=3$ rather than $\hat{d}=2$ [cf. Eqs. (3)] and the preaveraging approximation is applied. However, the qualitative behavior of $G(t)$ is not affected by that difference, therefore, the focus of the present article is on the influence of the rotational relaxation time on the FCS correlation function.

For the analysis of the FCS correlation function, the same parameters as in the previous section (Sec. V A) are used. Figure 2 displays FCS correlation functions for the polymer lengths $L=0.5,1,2,5$, and $10 \mu \mathrm{m}$. This length range corresponds to $p L=0.005-0.1$, well in the rodlike regime. Correlation functions [Eq. (47)] with the full expression for $\Phi$ [Eq. (46)] are presented, as well as correlation functions comprising the intramolecular dynamics only, i.e., the term $6 D t$ is omitted. An increase of the polymer length leads to a shift of the correlation function to large times (at least for the considered lengths). The reason is that the center-of-mass motion of the polymer contributes significantly to the decay of the correlation function, which is evident from the dotted lines. Since the diffusion coefficient $D$ [Eq. (31)] decreases with increasing length, and the correlation function decays when the polymer mean square displacement is on the order of $r_{0}^{2}$, i.e., $\left\langle\Delta r^{2}\right\rangle \approx r_{0}^{2}$ [cf. Eq. (49)], the characteristic decay time is proportional to $1 / D$ and therefore increases with the polymer length. The behavior changes as soon as the intramolecular dynamics dominates the mean square displacement. First, the rotational diffusion yields a significant contribution, because it increase as $L^{2}$ [Eq. (39)]. This applies as long as $t \gtrsim \widetilde{\tau}_{1}$ [Eq. (37)]. Since $\widetilde{\tau}_{1}$ increase very strongly with the polymer length $\left(\sim L^{3}\right)$, the

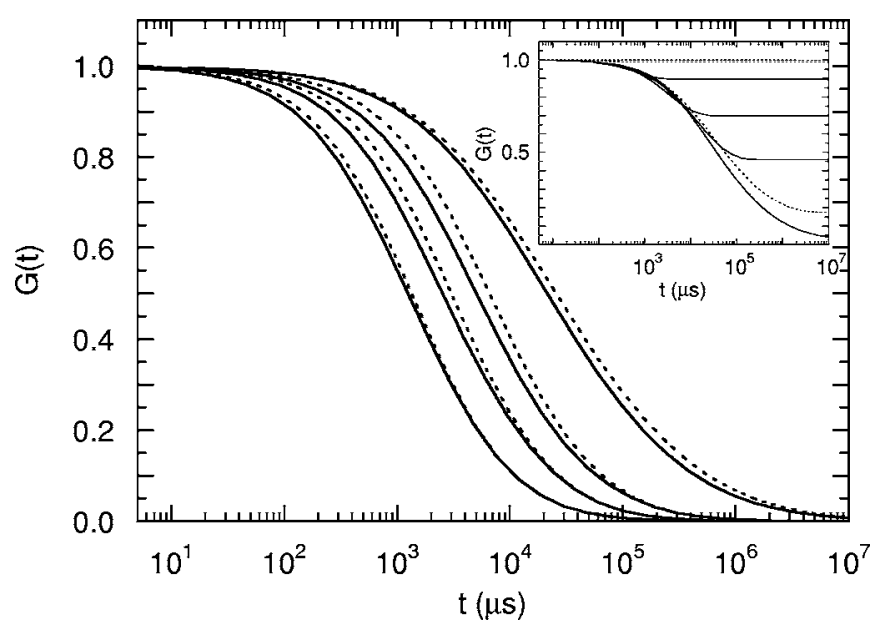

FIG. 3. FCS correlation functions for semiflexible polymers of various lengths: $L=0.5,1,2$, and $20 \mu \mathrm{m}$ (from left to right). The solid lines are determined including all internal modes, whereas for the dotted lines the first mode (rotational motion) is suppressed. The inset shows the same comparison without center-of-mass motion (length increases from top to bottom). The two shortest lengths yield $G(t)=1$ and are hence indistinguishable in the inset.

rotational diffusion exhibits ultimately the same length dependence as the diffusion coefficient $D$ on time scales $t$ $\ll \widetilde{\tau}_{1}$. Thus, for a very long rod, the sum of the rotational and translational diffusion coefficient will contribute to the decay of the correlation function. Only for $t>\widetilde{\tau}_{1}$, the translation diffusion will be measured. The contribution of the bending modes increases very slowly with $L$, namely, as $(\ln L / d)^{3 / 4}$ [Eq. (39)]. It dominates the correlation function when the center-of-mass mean square displacement together with the rotational contribution is much smaller than the intramolecular mean square displacement, which requires very long rodlike polymers, and applies for times $t \ll r_{0}^{2} /\left(6 D+L^{2} D_{r} / 3\right)$.

Figure 3 shows FCS correlation functions with (solid lines) and without (dotted lines) the contribution of the rotational motion for the polymer lengths $L=0.5,1,2$, and $20 \mu \mathrm{m}$. There is a pronounced influence of the rotational motion on the decay of the correlation function for this length range. For $L=0.5 \mu \mathrm{m}$, the bending modes do not contribute to the decay of the correlation function; their contribution is even negligible for $L=1 \mu \mathrm{m}$. This is evident from the correlation functions evaluated with the bending modes only (inset) - they are almost identical to unity for $L$ $=0.5 \mu \mathrm{m}$ and $L=1 \mu \mathrm{m}$. Here, the difference between the curves with and without the first mode directly reflects the influence of the rotational motion. With increasing polymer length, higher modes gain more weight. For $L=20 \mu \mathrm{m}$, bending modes already contribute significantly to the decay of the correlation function. As discussed above, for even longer polymers, bending modes will solely determine the shape of $G(t)$. As displayed in the figure, the rotational motion affects $G(t)$ at short times for short polymers and shifts to longer times with increasing polymer length. Hence, in a certain range of polymer lengths, the rotational diffusion coefficient can be determined from the FCS correlation function. For the considered parameters, the length range covers $L \approx 0.5-50 \mu \mathrm{m}$. Since various biological semiflexible poly- 


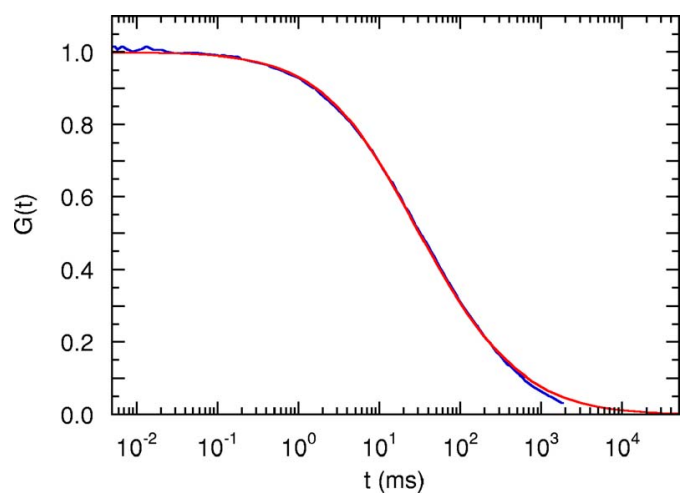

FIG. 4. (Color online) FCS correlation function of actin filaments. The scattered curve is taken from the experimental results presented in Ref. 15 and the smooth line has been calculated using Eqs. (46) and (47). The filament length is $L=20 \mu \mathrm{m}, l_{p}=17 \mu \mathrm{m}$, and the thickness $d=7 \mathrm{~nm}$.

mers such as actin filaments or fd viruses are of that length range, FCS opens the possibility to measure their rotational dynamics.

\section{Comparison with experimental results}

In Ref. 15, experimental measurements of the FCS correlation function and the extracted mean square displacement of fully labeled actin filaments are presented. To determine molecular parameters, an analytical expression for the mean square displacement derived in Refs. 25 and 26 is used and fitted to the mean square displacement. Since this expression uses a simplification for the mode number dependence valid for large modes only and does, strictly speaking, not include the rotational motion, a comparison with results derived in the current article will shed light on the relevance of long relaxation times (small mode numbers) for the decay of the FCS correlation function.

The parameters of the last sections are adopted to those of actin filaments, i.e., the persistence length $l_{p}=17 \mu \mathrm{m}$ and the thickness $d=7 \mathrm{~nm}$ are used. The parameters of the experimental setup are $r_{0}=0.21 \mu \mathrm{m}$ and $f=5.24 .{ }^{15}$

Here, the following strategy is followed to gain molecular parameters from the experimental data. The correlation function [Eq. (47)] is adjusted to the experimental results using two parameters, the length $L$ of the polymer and a factor $\Lambda$ adjusting the time scale. The latter factor simultaneously adjusts the diffusion coefficient and the relaxation times. Then, this function is compared with the analytical expression (49) to test the validity of the applied approximations.

The experimental results, taken from Ref. 15 for the homogeneously labeled actin filaments, are presented in Fig. 4 together with the correlation function [Eq. (47)] determined by the full solution of the eigenvalue problem. (For normalization, the experimental data for $G$ are divided by $2.75 \times 10^{-7} \mathrm{cps}^{2}$.) Good agreement is obtained for $L$ $\approx 20 \mu \mathrm{m}(p L=0.58)$ and $\Lambda=4 / 5$. Only for $t>10^{2} \mathrm{~ms}$ is the experimental correlation function smaller than the theoretical $G(t)$.

Figure 5 displays the numerically determined FCS correlation and the analytical approximation [Eq. (49)] with $\left\langle\Delta \boldsymbol{r}^{2}\right\rangle$ determined via Eq. (27). The approximation repro-

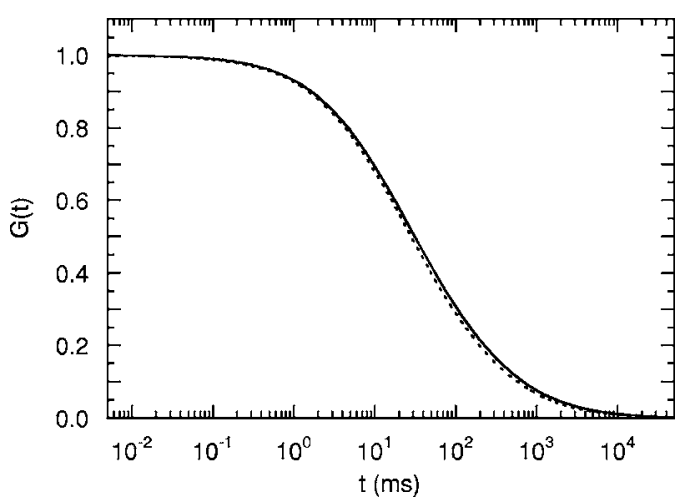

FIG. 5. Comparison of the exact theoretical FCS correlation function of actin filaments (solid line) with the analytical approximation (49) (dotted line).

duces the exact expression rather well; however, there is a shift to smaller times by approximately $10 \%$ in the visible time window. In the limit $t \rightarrow \infty$, both functions are equal. This shift becomes smaller for stiffer polymers.

With the length $L=20 \mu \mathrm{m}$, the mean square displacement [Eq. (27)] is calculated and compared with the experimental data of Ref. 15 in Fig. 6. Good agreement is obtained up to $t \approx 0.5 \mathrm{~s}$. This verifies the procedure applied in Ref. 15 . However, in order to match the theoretical and experimental time scales, I divided the experimental scale by 1.7 . The reason is not evident to me, because the FCS correlation function determined with the same mean square displacement reproduces the full correlation function well (cf. Fig. 5) with a scale factor of only 1.1 .

The theoretical correlation function provides the following estimates of the diffusion coefficient and rotational relaxation time: $D \approx 0.23 \mu \mathrm{m}^{2} / \mathrm{s}$ and $\widetilde{\tau}_{1} \approx 87 \mathrm{~s}$. The latter yields the rotational diffusion coefficient $D_{\mathrm{r}}=1 / 2 \widetilde{\tau}_{1} \approx 0.0057 \mathrm{~s}^{-1}$. For a rod, the expression for the translational diffusion coefficient: $D_{\mathrm{tr}}=k_{B} T[\ln (L / d)+0.32] /(3 \pi \eta L)$ yields the (spacial averaged) value $D_{\mathrm{tr}} \approx 0.18 \mu \mathrm{m}^{2} / \mathrm{s}$, which is approximately $20 \%$ smaller than the value extracted from the measurements. The rotational diffusion coefficient follows as $D_{\text {rr }}$ $=1 / 2 \tau_{\mathrm{rr}}=0.0036 \mathrm{~s}^{-1}$ [Eq. (53)] and is approximately $45 \%$ smaller. The increase in the diffusion coefficients is expected

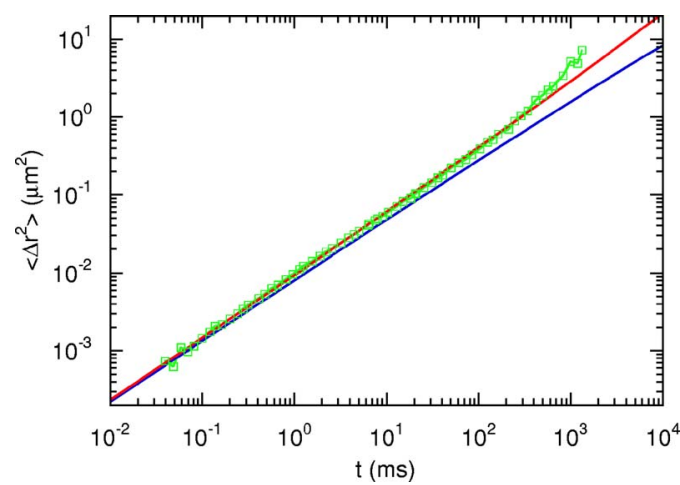

FIG. 6. (Color online) Average mean square displacement [Eq. (27)] of actin filaments. The top solid line is the theoretical result of the total mean square displacement and the bottom line is the mean square displacement in the center-of-mass reference frame. The symbols are experimental values taken from Ref. 15. Note, the time scale of the experiment is devided by 1.7 . 
for semiflexible polymers, since the longest relaxation time decreases with decreasing persistence length and the diffusion coefficient increases with increasing flexibility. ${ }^{17} \mathrm{Nev}-$ ertheless, the long time motion of the actin filaments agrees reasonably well with the dynamics of a rod.

The analysis reveals a strong influence of the rotational relaxation time on the decay of the correlation function. The quantitative difference is similar to that in Fig. 3 for the length $L=20 \mu \mathrm{m}$. For $t \ll \widetilde{\tau}_{1}$, the contribution of the rotational motion to the mean square displacement is $L^{2} D_{\mathrm{r}} / 3$ $\approx 0.8 \mu \mathrm{m}^{2} / \mathrm{s}$, which is rather close to the center-of-mass contribution $6 D \approx 1.4 \mu \mathrm{m}^{2} / \mathrm{s}$ and hence cannot be neglected. These figures suggest that the bare intramolecular motion should be visible for $t \ll 20 \mathrm{~ms}$. Figure 6 confirms the estimate and exhibits the dominance of the bending modes for $t<0.1 \mathrm{~ms}$.

The length of the actin filaments is used as a fit parameter. However, this length is not well defined, since actin filaments are living polymers, which grow or shrink in the course of time. In Ref. 15, the length of the actin filaments has been measured by fluorescence microscopy which gave lengths in the range of 4-8 $\mu \mathrm{m}$. The extracted length overestimates these values by a factor of $2-5$. Similarly, the length $L \approx 2.25 \mu \mathrm{m}$ determined in Ref. 15 underestimates the length by a similar factor. The difference between the experimental FCS correlation function and the theoretical curve in Fig. 4 for $t>0.1 \mathrm{~s}$ could be related to changes in the filament length; the smaller experimental value would be consistent with a shorter polymer length. Thus, for a basic understanding of the dynamics of rodlike polymers, it is desirable to measure the dynamics of well-defined samples, which do not change in length or any other property during the FCS experiment. Only then, the validity of one or the other theoretical model can unambiguously be verified.

Finally, I would like to comment on a few aspects.

The analytical expression for the correlation function derived in Ref. 15 [Eq. (20)] applies in the limit $L \rightarrow \infty$, as clearly said there. As discussed in Sec. IV, Eq. (49) suggest that this limiting behavior applies as long as $\left\langle\Delta r(t)^{2}\right\rangle \ll L^{2}$ $+3 f^{2} r_{0}^{2} / 2$. The parameters of that paper imply $\left\langle\Delta \boldsymbol{r}(t)^{2}\right\rangle$ $\ll 7 \mu \mathrm{m}$. Thus, the mean square displacements at large times might not be accurate anymore. The situation is different for a filament length of $L=20 \mu \mathrm{m}$, where $\left\langle\Delta r(t)^{2}\right\rangle \ll 400 \mu \mathrm{m}$.

The difference in the polymer length $-L=2.25 \mu \mathrm{m}$ in Ref. 15 and $L=20 \mu \mathrm{m}$ in the current article-is to a less extent related to the neglect of the rotational motion (no strict separation of rotation and bending modes is made in Ref. 15) than to the treatment of the mode numbers in general. As is well known, ${ }^{3,49}$ the wave numbers of a weakly bending rod exhibit the dependence $\zeta_{n}=(2 n-1) \pi / 2 L$ $(n>1)$ [Eq. (17)] and not $\zeta_{n}=n \pi / L$. In Ref. 15, the latter relation is used. The difference does not matter as long as only large mode numbers are considered, which is the case for time scales $t \ll \widetilde{\tau}_{2}$, i.e., time scales where the intramolecular dynamics dominates the mean square displacement. ${ }^{25,26}$ However, for not too long polymers, the decay of the FCS correlation is determined by relaxation times with small mode numbers, in particular, the rotational relaxation time and the center-of-mass motion. Here, the difference matters because of the strong dependence of the relaxation times on $\zeta_{n}[$ Eq. (17)].

Another aspect is the appropriate diffusion coefficient $D$. The spacial average [Eq. (28)] is used in the current article. As soon as the center-of-mass motion contributes to the decay of the FCS correlation function, its transverse and parallel components have to be taken into account, because they differ by only a factor of 2 [Eq. (29)].

\section{CONCLUSIONS}

The dynamics and the FCS correlation function have been theoretically described for weakly bending polymers. Particular attention has been paid to the influence of the rotational motion on the decay of the correlation function. Moreover, an analytical expression has been derived which relates the FCS correlation function to the segmental mean square displacement independent of any particular model for the polymer dynamics.

The applied Gaussian semiflexible polymer model includes the bending modes as well as the rotational dynamics in a coherent manner and thus yields consistent normal mode amplitude correlation functions in contrast to previous weakly bending rod models. ${ }^{49-51}$ The detailed analysis exhibits a strong influence of the rotational motion on the time dependence of the FCS correlation function for a certain range of polymer lengths. Since actin filaments or fd viruses are within that length scale, FCS provides the opportunity to measure their rotational diffusion coefficient. The bending dynamics, on the other hand, is only determining the initial decay of the correlation function and is hence difficult to characterize quantitatively in that length range. A comparison of the theoretical FCS curve with experimental results on actin filaments ${ }^{15}$ exhibits good agreement and the extracted translational and rotational diffusion coefficients are close to those of a rod. Since the relaxation times for small mode numbers determine the decay of the correlation function, the dependence of the wave numbers $\zeta_{n}$ on $n$ has to be taken into account adequately.

As is apparent, a semiflexible polymer model is required to determine the molecular parameters. The FCS correlation function itself or the extracted mean square displacement, e.g., via Eq. (49), can be fitted by the model. Various assumptions are required to derive the analytical expression relating the correlation function to the mean square displacement of a multilabeled polymer. Thus, we prefer and recommend to directly fit the model to the FCS correlation function. In contrast, for a single labeled polymer, the segmental mean square displacement is easily obtained. ${ }^{13,14,16}$

Actin filaments grow or shrink during an FCS experiment and their length is not well defined. Experimental studies of well-defined weakly bending rod systems are required to provide a standard. Only such a standard allows for an unambiguous verification of a particular theoretical model, which is necessary to elucidate its ability to correctly yield the desired molecular parameters. It is hoped that the current theoretical studies will stimulate such measurements. 
${ }^{1}$ P. Janmey, Handbook of Biological Physics (North-Holland, Amsterdam, 1995), Vol. 1A.

${ }^{2}$ M. A. Tracy and R. Pecora, Annu. Rev. Phys. Chem. 43, 525 (1992).

${ }^{3}$ L. Harnau, R. G. Winkler, and P. Reineker, J. Chem. Phys. 102, 7750 (1995).

${ }^{4}$ S. B. Smith, L. Finzi, and C. Bustamante, Science 258, 1122 (1992).

${ }^{5}$ C. Bustamante, J. F. Marko, E. D. Siggia, and S. Smith, Science 265 1599 (1994)

${ }^{6}$ T. T. Perkins, D. E. Smith, R. G. Larson, and S. Chu, Science 268, 83 (1995).

${ }^{7}$ J. F. Marko and E. D. Siggia, Macromolecules 28, 8759 (1995).

${ }^{8}$ S. R. Quake, H. Babcock, and S. Chu, Nature (London) 388, 151 (1997).

${ }^{9}$ R. G. Winkler, Phys. Rev. Lett. 82, 1843 (1999).

${ }^{10}$ R. G. Winkler, J. Chem. Phys. 118, 2919 (2003).

${ }^{11}$ E. P. Petrov and P. Schwille, State of the Art and Novel Trends in Fluoresence Correlation Spectroscopy, Methods and Applications of Fluorescence (Springer, Berlin, 2007).

${ }^{12}$ D. Lumma, S. Keller, T. Vilgis, and J. O. Rädler, Phys. Rev. Lett. 90, 218301 (2003).

${ }^{13}$ R. Shusterman, S. Alon, T. Gavrinyov, and O. Krichevsky, Phys. Rev. Lett. 92, 048303 (2004).

${ }^{14}$ R. G. Winkler, S. Keller, and J. O. Rädler, Phys. Rev. E 73, 041919 (2006).

15 A. Bernheim-Groswasser, R. Shusterman, and O. Krichevsky, J. Chem. Phys. 125, 084903 (2006).

${ }^{16}$ E. P. Petrov, T. Ohrt, R. G. Winkler, and P. Schwille, Phys. Rev. Lett. 97, 258101 (2006).

${ }^{17}$ L. Harnau, R. G. Winkler, and P. Reineker, J. Chem. Phys. 104, 6355 (1996).

${ }^{18}$ B. H. Zimm, J. Chem. Phys. 24, 269 (1956).

${ }^{19}$ M. Doi and S. F. Edwards, The Theory of Polymer Dynamics (Clarendon, Oxford, 1986)

${ }^{20}$ R. G. Winkler, L. Harnau, and P. Reineker, Macromol. Theory Simul. 6, 1007 (1997)

${ }^{21}$ G. Allegra and F. Ganazzoli, J. Chem. Phys. 74, 1310 (1981).

${ }^{22}$ E. Farge and A. C. Maggs, Macromolecules 26, 5014 (1993).

${ }^{23}$ R. Götter, K. Kroy, E. Frey, M. Bärmann, and E. Sackmann, Macromolecules 29, 30 (1996).

${ }^{24}$ L. Harnau, R. G. Winkler, and P. Reineker, J. Chem. Phys. 106, 2469 (1997).

${ }^{25}$ R. Granek, J. Phys. II 7, 1761 (1997).

${ }^{26}$ K. Kroy and E. Frey, Phys. Rev. E 55, 3092 (1997).

${ }^{27}$ D. C. Morse, Phys. Rev. E 58, R1237 (1998).

${ }^{28}$ F. Gittes and F. C. McKintosh, Phys. Rev. E 58, R1241 (1998).

${ }^{29}$ Since semiflexible polymers are comprised of atoms, the thermal fluctuations among noncovalently bound neighboring atoms lead to fluctuations of the contour length.

${ }^{30}$ O. Kratky and G. Porod, Recl. Trav. Chim. Pays-Bas 68, 1106 (1949).

${ }^{31}$ R. A. Harris and J. E. Hearst, J. Chem. Phys. 44, 2595 (1966).

${ }^{32}$ K. F. Freed, Adv. Chem. Phys. 22, 1 (1972).

${ }^{33}$ M. G. Bawendi and K. F. Freed, J. Chem. Phys. 83, 2491 (1985).
${ }^{34}$ S. M. Battacharjee and M. Muthukumar, J. Chem. Phys. 86, 411 (1987).

${ }^{35}$ J. B. Langowski, J. Noolandi, and B. Nickel, J. Chem. Phys. 95, 1266 (1991).

${ }^{36}$ A. M. Gupta and S. F. Edwards, J. Chem. Phys. 98, 1588 (1993).

${ }^{37}$ M. Otto, J. Eckert, and T. A. Vilgis, Macromol. Theory Simul. 3, 543 (1994).

${ }^{38}$ B. Y. Ha and D. Thirumalai, J. Chem. Phys. 103, 9408 (1995).

${ }^{39}$ R. G. Winkler and P. Reineker, Macromolecules 25, 6891 (1992).

${ }^{40}$ R. G. Winkler, P. Reineker, and L. Harnau, J. Chem. Phys. 101, 8119 (1994).

${ }^{41}$ F. Valle, M. Favre, P. D. L. Rios, A. Rosa, and G. Dietler, Phys. Rev. Lett. 95, 158105 (2005).

${ }^{42}$ M. Salomo, K. Kegel, C. Gutsche, M. Struhalla, J. Reinmuth, W. Skokow, U. Hahn, and F. Kremer, Colloid Polym. Sci. 284, 1325 (2006).

${ }^{43}$ H. Daniels, Proc. R. Soc. Edinburgh, Sect. A: Math. Phys. Sci. 63, 290 (1952).

${ }^{44}$ J. Wilhelm and E. Frey, Phys. Rev. Lett. 77, 2581 (1996).

${ }^{45} \mathrm{H}$. Kleinert, Path Integrals in Quantum Mechanics, Statistics, and Polymer Physics (World Scientific, Singapore, 1990).

${ }^{46}$ J. Samuel and S. Sinha, Phys. Rev. E 66, 050801 (2002).

${ }^{47}$ D. J. Bicout and T. W. Burkhardt, J. Phys. A 34, 5745 (2001).

${ }^{48}$ L. Le Goff, O. Hallatschek, E. Frey, and F. Amblard, Phys. Rev. Lett. 89, 258101 (2002).

${ }^{49}$ S. R. Aragón and R. Pecora, Macromolecules 18, 1868 (1985).

${ }^{50}$ S. R. Aragón, Macromolecules 24, 3451 (1991).

${ }^{51}$ S. R. Aragón, Macromolecules 20, 370 (1987).

${ }^{52}$ J. K. G. Dhont, An Introduction to Dynamics of Colloids (Elsevier, Amsterdam, 1996).

${ }^{53}$ H. Risken, The Fokker-Planck Equation (Springer, Berlin, 1989).

${ }^{54}$ S. Fujime and T. Maeda, Macromolecules 18, 191 (1985).

${ }^{55} \mathrm{~J}$. Howard, Mechanics of Motor Proteins and the Cytoskeleton (Sinauer Assoc., Sunderland, 2001).

${ }^{56}$ D. Magde, E. Elson, and W. W. Webb, Phys. Rev. Lett. 29, 705 (1972).

${ }^{57}$ E. Elson and D. Magde, Biopolymers 13, 1 (1974).

${ }^{58}$ M. Eigen and R. Rigler, Proc. Natl. Acad. Sci. U.S.A. 91, 5740 (1994).

${ }^{59}$ E. Haustein and P. Schwille, Methods 29, 153 (2003).

${ }^{60}$ N. L. Thompson, A. M. Lieto, and N. W. Allen, Curr. Opin. Struct. Biol. 12, 634 (2002).

${ }^{61}$ O. Krichevsky and G. Bonnet, Rep. Prog. Phys. 65, 251 (2002).

${ }^{62}$ D. Magde, E. L. Elson, and W. Webb, Biopolymers 13, 29 (1974).

${ }^{63}$ P. Schwille, Cell Biochem. Biophys. 34, 383 (2001).

${ }^{64}$ R. Rigler, U. Mets, J. Widengren, and P. Kask, Eur. Biophys. J. 22, 169 (1993).

${ }^{65}$ J. Rička and T. Binkert, Phys. Rev. A 39, 2646 (1989).

${ }^{66}$ For a function which depends on $\left|s-s^{\prime}\right|$.

${ }^{67}$ H. Maeda and N. Saitô, J. Phys. Soc. Jpn. 27, 984 (1969).

${ }^{68}$ J. M. Rallison and L. G. Leal, J. Chem. Phys. 74, 4819 (1981)

${ }^{69}$ J. Wilcoxon and J. M. Schurr, Biopolymers 22, 849 (1983).

${ }^{70}$ M. Schmidt and W. H. Stockmayer, Macromolecules 17, 509 (1984).

${ }^{71}$ K. Kubota, H. Urabe, and Y. Tominaga, Macromolecules 17, 2096 (1984). 